\title{
Self-reported muscle pain in adolescents with migraine and tension-type headache
}

Cephalalgic

32(3) 24I-249

(C) International Headache Society 2012

Reprints and permissions:

sagepub.co.uk/journalsPermissions.nav DOI: I0.1 I77/0333।024||434808 cep.sagepub.com

@SAGE

\author{
Astrid Blaschek', Astrid Milde-Busch ${ }^{2}$, Andreas Straube ${ }^{3}$, \\ Christoph Schankin ${ }^{3}$, Thyra Langhagen ${ }^{4}$, Klaus Jahn ${ }^{3,4}$, \\ Andreas Sebastian Schröder ${ }^{1,4}$, Karl Reiter ${ }^{5}$, Rüdiger von Kries ${ }^{2}$ \\ and Florian Heinen ${ }^{1,4}$
}

\begin{abstract}
Aim: To identify possible associations between muscular pain and headache in adolescents in a large population-based sample.

Methods: Grammar school students were invited to fill in a questionnaire on headache and associated lifestyle factors. Headache was classified according to the German version of the International Classification of Headache Disorders (2nd edition). Muscular pain was assessed via denoting affected areas in schematic drawings of a body and via provoked muscular pain on controlled movements of head, neck and shoulder regions.

Results: Prevalence of any headache within the previous 6 months exceeded $80 \%$. In all subjects muscular pain or pain on movement was most prominent in the neck and shoulder region, ranging from $9 \%$ to $27 \%$ in the non-headache population to up to $63 \%$ for individuals with migraine or mixed migraine and tension-type headache (TTH). Frequency of muscular pain increased significantly with growing chronicity of TTH.

Interpretation: A strong association between muscle pain in the neck/shoulder region and headache was observed, pointing to the importance of muscular pain for headache in adolescents. Also, in this age group muscular pain appears to be of particular importance in chronic TTH and - unexpectedly - in migraine, which is the most important new finding in our study.
\end{abstract}

\section{Keywords}

Muscle pain, headache, migraine, tension-type headache, adolescents

Date received: 3I July 20 I I; revised: 3 October 20 II; accepted: 10 December 20 I I

\section{Introduction}

Headache is recognised as a significant health complaint amongst adolescents (1). Migraine and tensiontype headache (TTH) are the most frequently reported primary headache syndromes in this age group, with an overall prevalence of headache within the 12 months before data collection of between 52 and $78 \%(2-4)$. In adults, muscular pain is known to be associated with both the intensity and the frequency of headache and to influence many outcome variables, e.g. severity of headache, burden of disease and chronicity (5-8). Based on these findings the EFNS task force on the treatment of TTH include a recommendation to examine the degree and location of pericranial muscle tenderness in a diagnostic workup for TTH, to assist with the choice of non-pharmacological treatment options (9). It is generally accepted that regular experience of
'Department of Paediatric Neurology and Developmental Medicine, Dr. von Hauner Children's Hospital, Ludwig-Maximilians-University Munich, Germany

${ }^{2}$ Institute of Social Paediatrics and Adolescent Medicine, LudwigMaximilians-University Munich, Germany

${ }^{3}$ Department of Neurology, Klinikum Grosshadern, Ludwig-MaximiliansUniversity Munich, Germany

${ }^{4}$ Integrated Center for Research and Treatment of Vertigo, Balance and Ocular Motor Disorders (IFBLMU), University Hospital Munich, Ludwig-Maximilians-University Munich, Germany

${ }^{5}$ Dr. von Hauner Children's Hospital, Ludwig-Maximilians-University Munich, Germany

Corresponding author:

Astrid Blaschek, Department of Paediatric Neurology and Developmental Medicine, Dr. von Hauner Children's Hospital, Ludwig-MaximiliansUniversity Munich, Lindwurmstrasse 4, 80337 Munich, Germany

Email: astrid.blaschek@med.uni-muenchen.de 
pain within the cervico-trigeminal loop results in sensitisation of midbrain pain structures (10) and becomes part of headache pathophysiology.

Epidemiological studies in children and adolescents have identified a whole variety of risk factors for headache $(3,4,11-13)$. These studies highlight the contribution of stress and lifestyle factors to any headache disorder, with a larger influence in migraineurs $(12,14)$. The role of muscular pain, however, has previously not been addressed in depth in these studies. The exact localisation of muscular pain in particular has not been assessed in large studies, although small-scale studies confined to highly selected patients point to the suspected importance of muscle tenderness for headache in children and adolescents (15-18). The studies suggest a high prevalence of concomitant neck pain contributing to the frequency, intensity and chronicity of any headache. However, there are conflicting data as to the contribution of muscular pain in migraineurs at present $(16,17)$. Such data are potentially helpful to define preventive strategies for headache and to impede its chronification when applied to adolescents.

As population-based studies on potential associations between muscular pain and headache are rare among adolescents $(11,19)$, the aim of the present study was to identify possible associations between headache and muscular pain in the pericranial, neck and shoulder muscles in a larger population-based sample. Applying a validated and previously published questionnaire allows classification of headache in order to evaluate differences concerning muscular pain between migraine and TTH $(2,20,21)$.

\section{Methods}

The study was approved by the Data Safety Officer and the Ethics Committee (082-08) of the Medical Faculty of the Ludwig-Maximilians-University Munich and the Bavarian State Ministry for Teaching and Culture. Students (grade 10 and 11, aged between 15 and 18 years) of 11 public grammar schools in Munich, Germany, were invited to fill in a questionnaire on headache and associated lifestyle factors. Recruitment procedures have been described in detail previously (14). Written informed consent was obtained from the parents of the participants. Consent of the participants themselves was assumed when they handed over the completed questionnaire to the study investigators. Students completed the questionnaire during a regular school lesson (45 minutes) in the classroom under supervision of a teacher and at least one member of the Munich headache team (medical doctor or psychologist) in a quiet and relaxed situation. Of all students present at school on the respective day of data collection, $94.8 \%$ agreed to fill in the questionnaire.

\section{Headache classification}

Subjects who responded positively to the screening question 'Did you have any headaches during the last seven days/three months/six months?' were classified as headache sufferers. They answered further questions regarding characteristics and symptoms as well as duration, frequency and intensity of their headaches. These questions were constructed according to the criteria laid down in the German version of the International Classification of Headache Disorders, 2nd edition (ICHD-II) (22). Based on these criteria, we used the following classification: 'pure migraine' (including the subtypes migraine with or without aura, chronic migraine, probable migraine and probable chronic migraine) and 'pure TTH' (including the subtypes infrequent episodic TTH, frequent episodic TTH, chronic TTH, probable infrequent episodic TTH, probable frequent episodic TTH and probable chronic TTH). A double diagnosis of 'migraine + TTH' could arise in subjects fulfilling the diagnostic criteria for both probable (chronic) migraine and probable (episodic or chronic) TTH, which requires compliance with all but one of the respective diagnostic criteria for migraine or TTH. All other subjects with headaches that did not match any of these diagnoses for primary headache were considered to have miscellaneous headache (MH). The presence of muscular pain was assessed in three ways: (1) Subjects who responded positively to the screening question 'Do you feel pain in the head, neck or shoulder?' were asked to mark these locations in each of three drawings with either frontal, back and lateral views of a body. (2) Subjects who responded positively to the screening question: 'Do you feel pain-sensitive spots in the head, neck or shoulder?' were asked to mark these spots in three further drawings with frontal, back and lateral views of a body. Based on these drawings, muscular pain and pain-sensitive spots in the following seven regions of interest were defined: frontal/temporal muscles, face, back of the head, neck, shoulder/neck, shoulder and chest. A grid subdividing each view into eight zones was used for analysis of data and defining regions of muscular pain or pain-sensitive spots. A mark encompassing adjacent zones counted for both. (3) Subjects were asked to respond to the following questions: 'Do you feel muscular pain in the head, neck or shoulder which could be associated with your headache during any of the following movements?' In this part students were given a detailed description of 13 movements and were asked to perform each of them. Supervision and assistance was provided as needed. The following movements were assessed: (i) head rotation to the left, (ii) head rotation to the right, (iii) head rotation to the left while looking up to the ceiling, (iv) head rotation to the right while 
looking up to the ceiling, (v) making a 'double chin', (vi) hyperextending the head to the back, (vii) tilting the head to one side, (viii) lowering down the shoulder, (ix) lowering down the shoulder while tilting the head to one side, (x) clenching the teeth, (xi) opening the mouth widely, (xii) bobbing the head, (xiii) other movements. These movements were categorized as follows: movements (i) to (iv) as rotation of the head, movements (v) to (vii) as movements of the neck, movements (viii) and (ix) as movements of the shoulder, movements (x) and (xi) as movement of the jaw joint, movement (xii) as rapid movements of the head and movement (xiii) as other movements.

\section{Statistical analyses}

Associations between categorical variables were tested by using chi-square statistics. Separate chi-square tests for any headache (with 'no headache' vs. 'any headache'), migraine as the only headache (with 'no headache' vs. 'pure migraine'), TTH as the only headache (with 'no headache' vs. 'pure TTH'), migraine + TTH (with 'no headache' vs. 'any migraine + any TTH') and MH (with 'no headache' vs. 'MH') were calculated. Furthermore, for subjects with TTH, CochraneArmitage tests for trend for ordered categories were calculated for infrequent episodic TTH, frequent episodic TTH and chronic TTH; both included probable and non-probable classifications. A similar analysis for 'chronicity' of migraine was not feasible due to low numbers of cases $(n=2)$. $P$-values $<0.05$ were considered to indicate a significant difference. Data were evaluated with the SAS software package (version 9.1, SAS Institute Inc., Cary, NC, USA).

\section{Results}

\section{Prevalence of headache}

A total of 1260 questionnaires were included in the analysis; $1047(83.1 \%)$ of the students reported headache at least once during the last 6 months. Amongst them, pure migraine was found in $129(10.2 \%)$ and pure TTH in $614(48.7 \%)$ of the participants. Of the 614 subjects with TTH, the following subtypes of TTH were found: $50(4.0 \%)$ subjects with infrequent episodic TTH, $75(6.0 \%)$ with frequent episodic TTH, 163 $(12.9 \%)$ with chronic TTH, $108(8.6 \%)$ subjects with probable infrequent episodic TTH, $101 \quad(8.0 \%)$ with probable frequent episodic TTH and $117(9.3 \%)$ with probable chronic TTH. Any type of migraine plus any TTH was reported by $249(19.8 \%)$ of the subjects. In the remaining $55(4.4 \%)$ subjects with headache, the type of headache could not be classified according to the International Headache Society (IHS) criteria.

\section{Muscular pain in head/neck and shoulder}

Localisation of muscular pain. Table 1 shows the proportion of subjects who marked specific regions of muscular pain in the head, neck or shoulder in the drawings, separately for subjects without headache, with any headache and stratified for type of headache. In all subjects muscular pain was most prominent in neck and shoulder region, ranging from 14 to $27 \%$ in the no headache population to up to $63 \%$ for individuals with migraine or mixed migraine and TTH headache. In subjects with pure TTH, the frequency of muscular pain ranged from 23 to $42 \%$, a trend towards higher frequency was observed in the chronic TTH group for muscular pain in the neck $(p=0.0029)$, shoulder/neck $(p=0.0003)$, shoulder $(p=0.0010)$ and the face region $(p=0.0393)$.

In addition, muscular pain in frontal, facial and chest muscles was more frequently reported by subjects with migraine or migraine and TTH than by individuals without headache.

Localisation of pain-sensitive spots. Table 2 shows the proportion of subjects who marked specific pain-sensitive spots in the head, neck or shoulder in the drawings, separately for subjects without headache, with any headache and stratified for type of headache. Painsensitive spots in the shoulder/neck region were by far the most reported. Around $17 \%$ of no headache adolescents reported pain-sensitive spots, the frequency in $\mathrm{TTH}$ nearly doubling up to $33 \%$ and tripling to around $50 \%$ in migraineurs or mixed migraine $+\mathrm{TTH}$.

Frequency of reporting increased statistically significantly with increasing chronicity of TTH for painsensitive spots in almost all regions of interest: frontal/temporal $(p<0.0001)$, face $(p=0.0010)$, back of the head $(p=0.0088)$, neck $(p=0.0102)$, shoulder/ neck $(p<0.0001)$ and shoulder $(p<0.0001)$, but not chest $(p=0.1140)$.

Pain-sensitive spots in the chest region were only reported in statistically significant numbers in students with migraine.

\section{Pain during movements in neck/shoulder region}

Table 3 shows the proportion of subjects who reported muscular pain that is potentially associated with specific movements, separately for subjects without headache, with any headache and stratified for type of headache. Movements were grouped into five main categories according to an anatomical basis. Taken together, significantly more subjects with any type of headache reported muscular pain along almost all tested movements. Stratified for types of headache, higher proportions were observed in students with 


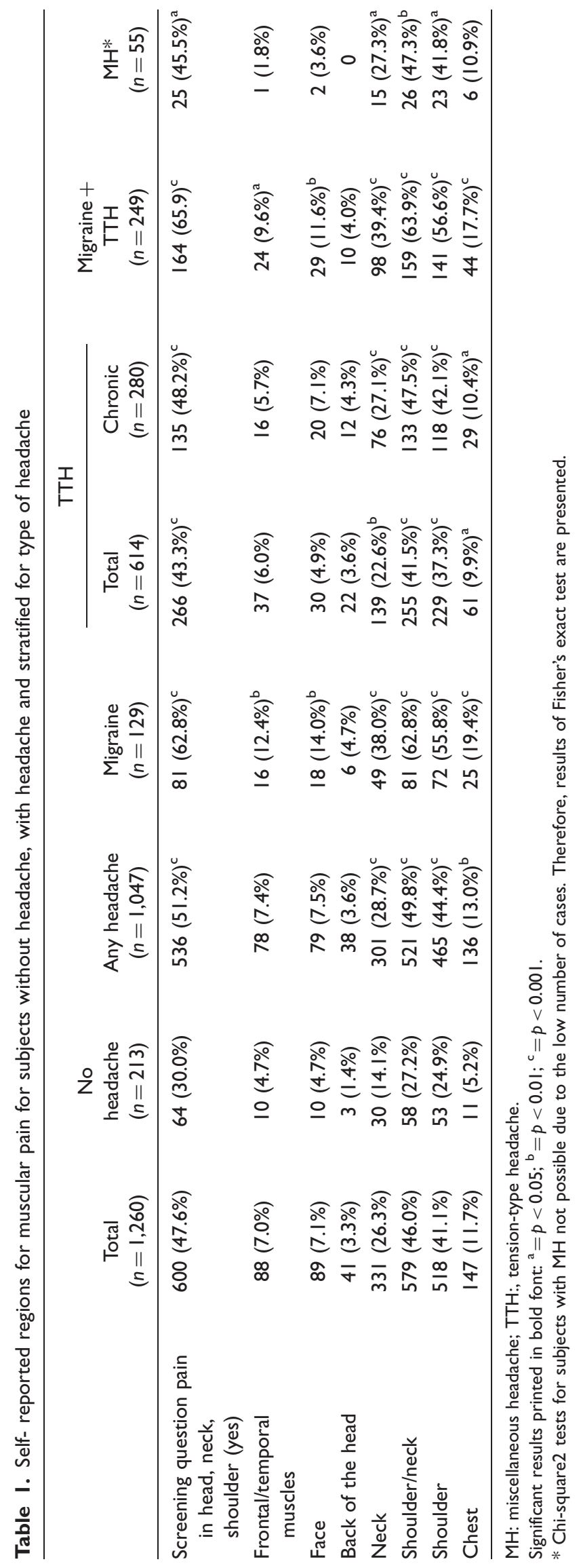




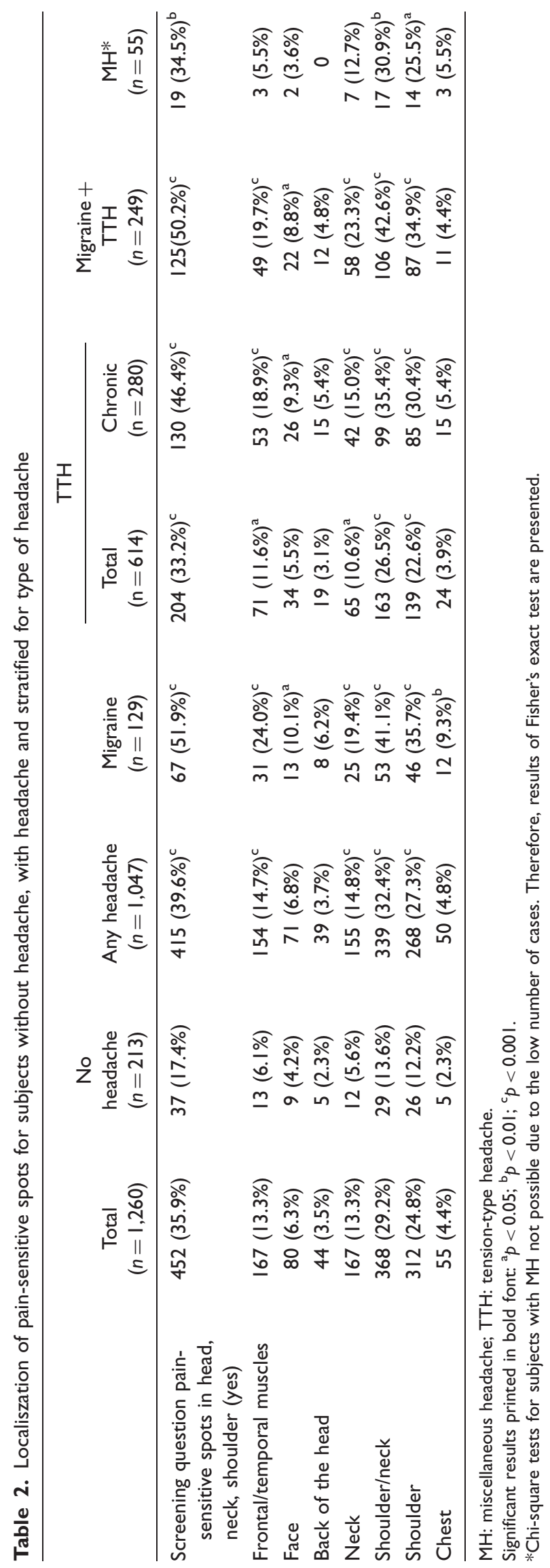




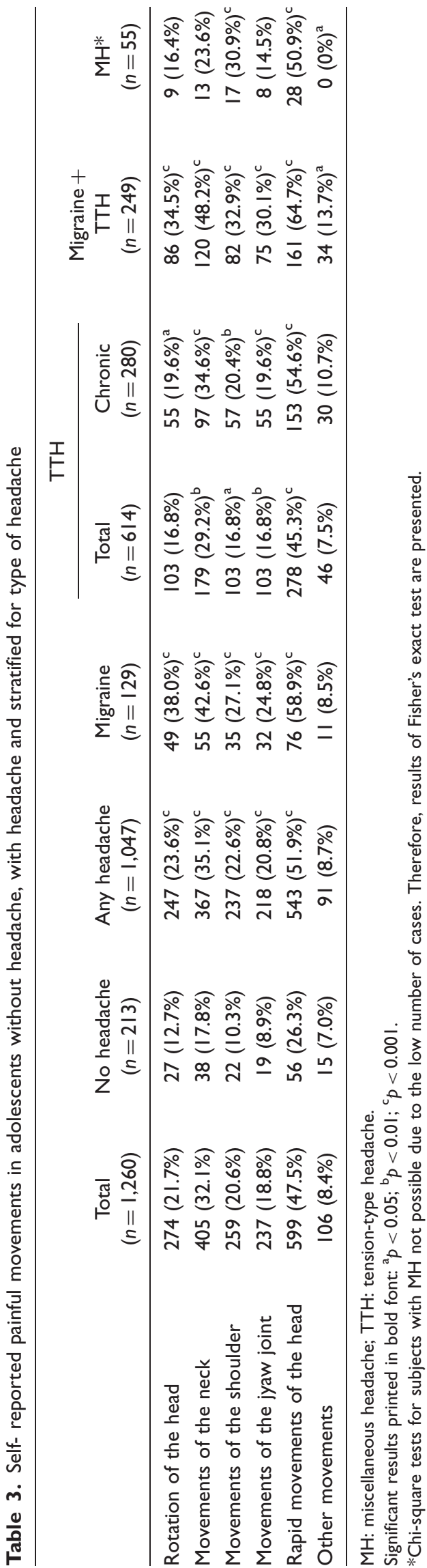

migraine $(25-59 \%)$ and combined migraine + TTH (33-65\%), this being equivalent to a two- to threefold increase when compared to individuals without any headache.

For subjects with TTH we observed increasing pain on movements with growing 'chronicity' of TTH: while individuals with infrequent episodic TTH reported only muscular pain associated with clenching the teeth $(16.5 \%)$ more frequently than students without headache $(6.1 \%)$ and subjects with frequent episodic TTH reported muscular pain associated when bobbing the head $(39.2 \%)$, subjects with chronic TTH reported muscular pain associated with six of the 13 tested movements more frequently than students without headache. Students with chronic TTH reported painful movements typically twice as often as students without headache. Furthermore, frequency of reporting increased statistically significantly with increasing chronicity of TTH for the following movements: rapid movement of the head $(p<0.0001)$, movement of the neck $(p=0.0082)$, movement of the shoulder $(p=0.0147)$, other movements $(p=0.0055)$, but not with rotation of the head $(p=0.0991)$ and movement of the jaw joint $(p=0.3215)$.

\section{Discussion}

In our large study group of generally healthy grammar school students the prevalence of any headache within the 6 months prior to data collection exceeded $80 \%$. This is in accordance with earlier studies conducted in Germany $(1,3)$ and in other countries around the world $(4,23)$. Furthermore, the prevalence of TTH and migraine were in good accordance to the results in these other epidemiological studies.

A remarkable difference in reporting muscular pain was observed between the students without headache and those experiencing headache: compared with the headache group, the proportion of students reporting muscular pain/pain-sensitive spots in the musculature or pain on movement was only about half in the no headache group.

The prevalence of reported muscular pain, painsensitive spots in the musculature or pain on movement increased along with the progression from episodic to chronic TTH and was highest among those with migraine.

These findings confirm earlier smaller studies in adolescents and adults showing higher frequencies of muscular pain in the shoulder/neck region in individuals with any type of headache $(11,16,17,24,25)$. This may reflect the shift to relevant changes within the cervicotrigeminal loop as one part of headache pathophysiology. A number of studies in adults have demonstrated a strong association between headache, cervical and thoracic spine dysfunction (5) and an increased tenderness 
to palpation of pericranial myofascial tissues (25). These studies suggest that increased tenderness is not only observed in TTH $(15,16)$ but also in migraine $(6)$.

The most accepted theory is that the central sensitisation process is a consequence of prolonged peripheral nociceptive inputs from muscle tissue within the cervico-trigeminal system (10). Most likely this mechanism is particularly relevant in patients with chronic myofascial pain, because inputs from muscle nociceptors are more effective in inducing prolonged changes in the behaviour of dorsal horn neurons (26). Bendtsen (10) proposed a model in which the main alteration in chronic headache involves sensitisation of dorsal horn/trigeminal nucleus neurons due to increased nociceptive inputs from pericranial myofascial tissues. In general it is thought that such a mechanism is responsible for the development of chronic TTH. The nociceptive input from myofascial A-delta- and C-fibres seems to be increased for several reasons (26), resulting in plastic changes in the spinal dorsal horn/trigeminal nucleus. As a consequence, the normal inhibitory effect of low threshold A-beta fibres on transmission in the spinal dorsal horn is altered to a pain stimulatory effect, potentiating the effect of nociceptive A-delta- and C-fibres (26). This increased nociceptive input to supraspinal structures may result in increased facilitation and decreased inhibition of pain transmission at the level of the spinal dorsal horn/trigeminal nucleus and in increased pericranial intramuscular activity. Mechanisms of central sensitisation and reduction of inhibitory mechanisms are probably both involved in altered central nociception in headache patients, thus inducing and maintaining the chronic pain condition (10). Along the process of chronification, sensitisation of pain pathways in the central nervous systems seem to be play an important part $(7,8)$. In this context it was not surprising that chronic TTH was associated with a higher prevalence of pain.

However, the above-mentioned theory of central sensitisation as a consequence of prolonged peripheral nociceptive input from intramuscular areas cannot explain easily the high prevalence of muscle pain in migraine patients. Most of these patients reported only episodic migraine attacks. The significantly increased co-occurrence of muscular pain in children with migraine might have several potential explanations. Studies of migraine reveal an increased sensitivity to observe and report other types of pain, probably resulting from the intensive experience of pain in migraine attacks (27). In a recent study by our group, children with migraine reported a considerable shift towards higher scores for perceived stress (14). Increased intramuscular activity secondary to psychological stress is likely to be relevant in this respect, because the stress condition may cause a prolonged increase in regional muscle tone via the limbic system and at the same time potentiate pain facilitation from the brain stem to the spinal dorsal horn (28). Moreover, neurophysiological studies in migraine disclose an abnormality of cortical excitability and information processing in patients with migraine between attacks. Lack of habituation is the principal and most reproducible interictal abnormality in sensory processing in these patients. Cortical habituation is seen as a protective mechanism against overstimulation or 'stress'. It is conceivable that stress could produce additional chemical changes in the cerebral cortex of sufficient degree to promote migraine attacks (28). It is not known if such a change of cortical excitability can also be induced due to an increased input from muscular nociceptors. In this context it is interesting that muscular trigger points are more often located in the ipsilateral pericranial muscles in patients with strict unilateral headaches (29). This finding still does not allow distinction between consequence and cause for migraine and trigger points. A further argument for a close relationship is the increased prevalence of chronic migraine (but also $\mathrm{TTH}$ ) in patients with fibromyalgia, the prototype of musculoskeletal pain disorder (30).

Given the known risk of neck pain to worsen the frequency of headache in adolescents and the fact that intramuscular tension is a significant risk factor for excess morbidity from cervical pain later on, our findings require further investigation to improve our understanding of the underlying pathophysiology (18). Considering the resultant negative impact on the adolescent's tertiary education and work participation in adulthood, proactive preventive strategies can be imagined to follow.

\section{Strength and limitations}

The major strength of our study is its population-based data collection, although recruitment was confined to a subgroup of students visiting a grammar school. Given the high participation rate $(94.8 \%)$ and the comparatively low number of excluded questionnaires (11.6\%), sampling bias seems to be unlikely. With the large number of participants, it was possible to differentiate between types of headache and to determine headachetype-specific associations between muscular pain and headache.

There are some weaknesses of the present study. Classifications of types of headache were not validated by physicians' diagnoses, but were based on a selfadministered questionnaire. This might have resulted in comparatively high estimates of headache prevalence, particularly for TTH. However, a number of studies found reasonable sensitivity, specificity and predictive values for headache screening questionnaires 
(31-33). The construction of the headache questionnaire was based on the criteria of the ICHD-II (e.g. 20,22). The questionnaire used was an established instrument to assess frequency and localisation of pain among children and adolescents and has been extensively used before $(2,21,34,35)$. Its suitability for the teenaged age group has also been demonstrated explicitly before $(20,34)$.

Localisation of pain/pain-sensitive spots was only self-reported, which may account for misclassification or failure to identify the 'true' pain-sensitive area identified by physical examination (for definition, see Methods). Validation of self-reported pain spots by physical examination was not possible because of the strictly anonymous data collection. However, former investigations have used comparable questionnaires $(11,19,24)$ or even shown a correlation between selfreported pain and results of clinical examination (e.g. 17,18). To come as close as possible within this given setting (by the Bavarian State Ministry for Teaching and Culture) to the regions of muscular pain we confirmed 'muscular pain' (i) by marking a sketch, (ii) by re-questioning for pain-sensitive spots and (iii) by pain on movement manoeuvers under supervision of one member of the Munich headache team.

The cross-sectional study design does not allow disentangling cause and effect by definition. Whether this pain hypersensitivity is a primary (cause) or a secondary (consequence) phenomenon is still under debate $(10,25)$.

In conclusion, a strong overall association between muscle pain in the neck/shoulder region and headache was observed. This is seen as confirmation of the importance of the cervico-trigeminal loop also in the spectrum of primary headache in adolescents. Showing that muscular pain appears to be particularly important not only for adolescents with chronic TTH but also for individuals with migraine is one new finding of our study.

\section{Acknowledgements}

The authors would like to thank Michaela Bonfert MD, Kristina Huß MD and Petra Sostak MD for giving lessons regarding headache in the participating schools. We thank all participants for their time and efforts. We also thank the contact persons at the participating schools for their effort in preparation of the data collection. We thank the Bavarian State Ministry for Teaching and Culture for understanding and support. The authors gratefully acknowledge the editorial assistance of Jörg Urban in the preparation of this manuscript.

\section{Funding}

This research received no specific grant from any funding agency in the public, commercial, or not-for-profit sectors.

\section{Conflict of interest}

The authors certify that they have no financial interest to disclose.

\section{References}

1. Ellert U, Neuhauser H and Roth-Isigkeit A. [Pain in children and adolescents in Germany: the prevalence and usage of medical services. Results of the German Health Interview and Examination Survey for Children and Adolescents (KiGGS)]. Bundesgesundheitsblatt Gesundheitsforschung Gesundheitsschutz 2007; 50: 711-717.

2. Fendrich K, Vennemann M, Pfaffenrath V, et al. Headache prevalence among adolescents - the German DMKG headache study. Cephalalgia 2007; 27: 347-354.

3. Kroner-Herwig B, Heinrich M and Morris L. Headache in German children and adolescents: a populationbased epidemiological study. Cephalalgia 2007; 27: 519-527.

4. Laurell K, Larsson B and Eeg-Olofsson O. Prevalence of headache in Swedish schoolchildren, with a focus on tension-type headache. Cephalalgia 2004; 24: 380-388.

5. Jull G, Trott P, Potter $\mathrm{H}$, et al. A randomized controlled trial of exercise and manipulative therapy for cervicogenic headache. Spine 2002; 27: 1835-1843. (discussion 43).

6. Blau JN and MacGregor EA. Migraine and the neck. Headache 1994; 34: 88-90.

7. Bendtsen L and Fernandez-de-la-Penas C. The role of muscles in tension-type headache. Curr Pain Headache Rep 2011; 15: 451-458.

8. Bezov D, Ashina S, Jensen R, et al. Pain perception studies in tension-type headache. Headache 2011; 51: 262-271.

9. Bendtsen L, Evers S, Linde M, et al. EFNS guideline on the treatment of tension-type headache - report of an EFNS task force. Eur J Neurol 2010; 17: 1318-1325.

10. Bendtsen L. Central sensitization in tension-type headache - possible pathophysiological mechanisms. Cephalalgia 2000; 20: 486-508.

11. Grimmer K, Nyland L and Milanese S. Repeated measures of recent headache, neck and upper back pain in Australian adolescents. Cephalalgia 2006; 26: 843-851.

12. Milde-Busch A, Blaschek A, Borggrafe I, et al. Associations of diet and lifestyle with headache in high-school students: results from a cross-sectional study. Headache 2010; 50: 1104-1114.

13. Milde-Busch A, von Kries R, Thomas S, et al. The association between use of electronic media and prevalence of headache in adolescents: results from a population-based cross-sectional study. BMC Neurol 2010; 10: 12.

14. Milde-Busch A, Blaschek A, Heinen F, et al. Associations between stress and migraine and tension-type headache: Results from a school-based study in adolescents from grammar schools in Germany. Cephalalgia 2011; 31: 774-785.

15. Fernandez-de-Las-Penas C, Fernandez-Mayoralas DM, Ortega-Santiago R, et al. Referred pain from myofascial trigger points in head and neck-shoulder muscles 
reproduces head pain features in children with chronic tension type headache. $J$ Headache Pain 2011; 12: 35-43.

16. Metsahonkala L, Anttila P, Laimi K, et al. Extracephalic tenderness and pressure pain threshold in children with headache. Eur J Pain 2006; 10: 581-585.

17. Anttila P, Metsahonkala L, Mikkelsson M, et al. Muscle tenderness in pericranial and neck-shoulder region in children with headache. A controlled study. Cephalalgia 2002; 22: 340-344.

18. Laimi K, Vahlberg T, Salminen J, et al. Does neck pain determine the outcome of adolescent headache? Cephalalgia 2007; 27: 244-253.

19. Smith L, Louw Q, Crous L, et al. Prevalence of neck pain and headaches: impact of computer use and other associative factors. Cephalalgia 2009; 29: 250-257.

20. Milde-Busch A, Heinrich S, Thomas S, et al. Quality of life in adolescents with headache: results from a population-based survey. Cephalalgia 2010; 30: 713-721.

21. Roth-Isigkeit A, Thyen U, Raspe HH, et al. Reports of pain among German children and adolescents: an epidemiological study. Acta Paediatr 2004; 93: 258-263.

22. Headache Classification Subcommittee of the International Headache Society. The International Classification of Headache Disorders: 2nd edition. Cephalalgia 2004; 24 (Suppl 1): 9-160.

23. Zwart JA, Dyb G, Holmen TL, et al. The prevalence of migraine and tension-type headaches among adolescents in Norway. The Nord-Trondelag Health Study (Head-HUNT-Youth), a large populationbased epidemiological study. Cephalalgia 2004; 24 : 373-379.

24. Anttila P, Metsahonkala L, Aromaa M, et al. Determinants of tension-type headache in children. Cephalalgia 2002; 22: 401-408.
25. Buchgreitz L, Lyngberg AC, Bendtsen L, et al. Increased pain sensitivity is not a risk factor but a consequence of frequent headache: a population-based follow-up study. Pain 2008; 137: 623-630.

26. Woolf CJ and Doubell TP. The pathophysiology of chronic pain - increased sensitivity to low threshold A beta-fibre inputs. Curr Opin Neurobiol 1994; 4: $525-534$.

27. Abu-Arafeh I and Russell G. Prevalence and clinical features of abdominal migraine compared with those of migraine headache. Arch Dis Child 1995; 72: 413-417.

28. Sauro KM and Becker WJ. The stress and migraine interaction. Headache 2009; 49: 1378-1386.

29. Fernandez-de-Las-Penas C, Cuadrado ML and Pareja JA. Myofascial trigger points, neck mobility and forward head posture in unilateral migraine. Cephalalgia 2006; 26 : 1061-1070.

30. de Tommaso M, Sardaro M, Serpino C, et al. Fibromyalgia comorbidity in primary headaches. Cephalalgia 2009; 29: 453-464.

31. Valentinis L, Valent F, Mucchiut M, et al. Migraine in adolescents: validation of a screening questionnaire. Headache 2009; 49: 202-211.

32. Zarifoglu M, Karli N and Taskapilioglu O. Can ID Migraine be used as a screening test for adolescent migraine? Cephalalgia 2008; 28: 65-71.

33. Hershey AD, Winner P, Kabbouche MA, et al. Use of the ICHD-II criteria in the diagnosis of pediatric migraine. Headache 2005; 45: 1288-1297.

34. Roth-Isigkeit A, Ellert U and Kurth BM. [Assessment of pain in The Child and Adolescent Health Survey]. Gesundheitswesen 2002; 64(Suppl 1): S125-S129.

35. Roth-Isigkeit A, Raspe $\mathrm{HH}$, Stoven $\mathrm{H}$, et al. [Pain in children and adolescents - results of an exploratory epidemiological study]. Schmerz 2003; 17: 171-178. 